\title{
SOME TRIPLE SERIES EQUATIONS INVOLVING JACOBI POLYNOMIALS
}

\author{
by J. S. LOWNDES
}

(Received September 8th 1967)

\section{Introduction}

Equations which may be regarded as extensions of the dual series equations discussed by Noble (1) and the present author (2) are the triple series equations of the first kind

$$
\begin{aligned}
& \sum_{n=0}^{\infty} C_{n} J_{n}(a, \lambda ; \rho)=g_{1}(\rho), \quad 0 \leqq \rho<d, \\
& \sum_{n=0}^{\infty} C_{n} p_{n}(\lambda-\sigma, \lambda) J_{n}(a, \lambda ; \rho)=f(\rho), \quad d<\rho<e, \\
& \sum_{n=0}^{\infty} C_{n} J_{n}(a, \lambda ; \rho)=h_{1}(\rho), \quad e<\rho \leqq 1,
\end{aligned}
$$

and the triple series equations of the second kind

$$
\begin{aligned}
& \sum_{n=0}^{\infty} D_{n} p_{n}(\lambda-\sigma, \lambda) J_{n}(a, \lambda ; \rho)=g(\rho), \quad 0 \leqq \rho<d, \\
& \sum_{n=0}^{\infty} D_{n} J_{n}(a, \lambda ; \rho)=f_{1}(\rho), \quad d<\rho<e, \\
& \sum_{n=0}^{\infty} D_{n} p_{n}(\lambda-\sigma, \lambda) J_{n}(a, \lambda ; \rho)=h(p), \quad e<\rho \leqq 1,
\end{aligned}
$$

where $f, f_{1}, g, g_{1}, h$ and $h_{1}$ are all known functions,

and

$$
p_{n}(\lambda-\sigma, \lambda)=\frac{\Gamma(\lambda-\sigma+n) \Gamma(1+a-\lambda+n)}{\Gamma(\lambda+n) \Gamma(1+a+\sigma-\lambda+n)},
$$

$$
J_{n}(a, \lambda ; \rho)={ }_{2} F_{1}(a+n,-n ; \lambda ; \rho),
$$

is the Jacobi polynomial (3).

If we write $a=\alpha+\beta+1, \lambda=\alpha+1, \rho=\sin ^{2} \theta / 2,0 \leqq \theta \leqq \pi$, and use the alternative definition of the Jacobi polynomial (4)

$$
P_{n}^{(\alpha, \beta)}(\cos \theta)=\frac{\Gamma(\alpha+1+n)}{\Gamma(n+1) \Gamma(\alpha+1)} J_{n}\left(\alpha+\beta+1, \alpha+1 ; \sin ^{2} \theta / 2\right),
$$

it can easily be shown that equations (1) to (6) reduce to the equations solved by Srivastava (5) when $\sigma=\frac{1}{2}$ and to those solved by Collins (6) when $\alpha=\beta=0$ and $\sigma=\frac{1}{2}$. 
In this paper we shall show that when $a+1>\lambda>\sigma, 0<\sigma<1$, the solution of the triple series equations can in each case be reduced to the solution of a Fredholm integral equation of the second kind. The method used is a generalisation of that used by Srivastava and is similar to the method employed by Cooke (7) to solve some triple integral equations. The general results are then applied to deduce an alternative form of the solution to that obtained by Collins for his equations. The analysis throughout is formal and in order to simplify the working we shall assume that $f_{1}=g_{1}=h_{1}=0$.

It will now be shown that once the solutions of the equations (1) to (6) are known for $a+1>\lambda>\sigma, 0<\sigma<1$, then it is an easy matter to obtain the solutions of the same equations when $a+1+\sigma>\lambda>0,-1<\sigma<0$.

Setting $\rho=1-\rho^{\prime}$ and using the result

$$
J_{n}\left(a, \lambda ; 1-\rho^{\prime}\right)=(-1)^{n} \frac{\Gamma(\lambda) \Gamma(1+a-\lambda+n)}{\Gamma(\lambda+n) \Gamma(1+a-\lambda)} J_{n}\left(a, 1+a-\lambda ; \rho^{\prime}\right),
$$

given in (1), we find that equations (1), (2) and (3) become

$$
\begin{aligned}
& \sum_{n=0}^{\infty} C_{n}^{\prime} p_{n}\left(\lambda^{\prime}-\sigma^{\prime}, \lambda^{\prime}\right) J_{n}\left(a, \lambda^{\prime} ; \rho^{\prime}\right)=h_{1}\left(1-\rho^{\prime}\right), 0 \leqq \rho^{\prime}<1-e, \\
& \sum_{n=0}^{\infty} C_{n}^{\prime} J_{n}\left(a, \lambda^{\prime} ; \rho^{\prime}\right)=f\left(1-\rho^{\prime}\right), 1-e<\rho^{\prime}<1-d, \\
& \sum_{n=0}^{\infty} C_{n}^{\prime} p_{n}\left(\lambda^{\prime}-\sigma^{\prime}, \lambda^{\prime}\right) J_{n}\left(a, \lambda^{\prime} ; \rho^{\prime}\right)=g_{1}\left(1-\rho^{\prime}\right), \quad 1-d<\rho^{\prime} \leqq 1,
\end{aligned}
$$

where

$$
C_{n}=(-1)^{n} \frac{\Gamma\left(\lambda^{\prime}\right) \Gamma\left(1+a-\lambda^{\prime}+n\right)}{\Gamma\left(\lambda^{\prime}+n\right) \Gamma\left(1+a-\lambda^{\prime}\right)} p_{n}\left(\lambda^{\prime}-\sigma^{\prime}, \lambda^{\prime}\right) C_{n}^{\prime}
$$

and $\lambda^{\prime}=1+a-\lambda, \sigma^{\prime}=-\sigma$.

Equations (11), (12) and (13) are triple series equations of the second kind whose solution can be found when $a+1>\lambda^{\prime}>\sigma^{\prime}, 0<\sigma^{\prime}<1$, and hence from equation (14) we can obtain the solution of equations (1), (2) and (3) when $a+1+\sigma>\lambda>0,-1<\sigma<0$. In a similar way equations (4), (5) and (6) can be transformed into equations of the first kind and their solution found when $a+1+\sigma>\lambda>0,-1<\sigma<0$.

We now give two results which will be required in the solution of the equations.

The orthogonality relation for Jacobi polynomials is

$$
\int_{0}^{1} r^{\lambda-1}(1-r)^{a-\lambda} J_{m}(a, \lambda ; r) J_{n}(a, \lambda ; r) d r=\frac{\delta_{m n}}{\Delta_{n}^{2}}, a+1>\lambda>0,
$$

where $\delta_{m n}$ is the Kronecker delta and

$$
\Delta_{n}^{2}=\frac{(a+2 n) \Gamma(a+n) \Gamma(\lambda+n)}{\Gamma(n+1)\{\Gamma(\lambda)\}^{2} \Gamma(1+a-\lambda+n)} .
$$


It is shown in (1) that when $a+1+\sigma>\lambda>\sigma>0$,

$$
\begin{aligned}
k(r, \rho) & =\{\Gamma(\sigma)\}^{2}(r \rho)^{\lambda-1} \sum_{n=0}^{\infty} \Delta_{n}^{2} p_{n}(\lambda-\sigma, \lambda) J_{n}(a, \lambda ; r) J_{n}(a, \lambda ; \rho) \\
& =\int_{0}^{t} m(x)(r-x)^{\sigma-1}(\rho-x)^{\sigma-1} d x=k_{t}(r, \rho),
\end{aligned}
$$

where $m(x)=x^{\lambda-\sigma-1}(1-x)^{\lambda-\sigma-a}$ and $t=\min (r, \rho)$.

\section{Equations of the first kind} we set

In order to solve the triple series equations of the first kind (with $g_{1}=h_{1}=0$ )

$$
\sum_{n=0}^{\infty} C_{n} J_{n}(a, \lambda ; \rho)=(1-\rho)^{\lambda-a} \phi(\rho), \quad d<\rho<e,
$$

and using the orthogonality relation (15) it follows from equations (1), (19) and (3) that

$$
C_{n}=\Delta_{n}^{2} \int_{d}^{e} r^{\lambda-1} \phi(r) J_{n}(a, \lambda ; r) d r, \quad a+1>\lambda>0,
$$

where $\Delta_{n}^{2}$ is defined by equation (16).

Substituting for $C_{n}$ into equation (2) and interchanging the order of integration and summation, we find

$$
\int_{d}^{e} \phi(r) k(r, \rho) d r=\{\Gamma(\sigma)\}^{2} \rho^{\lambda-1} f(\rho), \quad d<\rho<e,
$$

where $k(r, \rho)$ is defined by equation (17).

Using the notation of equation (18) we can write the above equation in the form

$$
\int_{d}^{\rho} \phi(r) k_{r}(r, \rho) d r+\int_{\rho}^{e} \phi(r) k_{\rho}(r, \rho) d r=\{\Gamma(\sigma)\}^{2} \rho^{\lambda-1} f(\rho),
$$

where $d<\rho<e$ and $a+1>\lambda>\sigma>0$.

Inverting the order of integration in equation (22) we get

$$
\int_{d}^{\rho} \frac{m(x)}{(\rho-x)^{1-\sigma}} \Phi(x) d x=\{\Gamma(\sigma)\}^{2} \rho^{\lambda-1} f(\rho)-\int_{0}^{d} \frac{m(x)}{(\rho-x)^{1-\sigma}} d x \int_{d}^{e} \frac{\phi(r)}{(r-x)^{1-\sigma}} d r,
$$

where

$$
\Phi(x)=\int_{x}^{e} \frac{\phi(r)}{(r-x)^{1-\sigma}} d r .
$$

Now equations (23) and (24) are Abel-type integral equations which may be solved when $0<\sigma<1$ to give

$$
\phi(r)=-\frac{\sin (\sigma \pi)}{\pi} \frac{d}{d r} \int_{r}^{e} \frac{\Phi(x)}{(x-r)^{\sigma}} d x, \quad d<r<e,
$$


and

$$
m(x) \Phi(x)=F(x)-\frac{\sin (\sigma \pi)}{\pi} \int_{0}^{d} m(\xi) l(x, \xi) d \xi \int_{d}^{e} \frac{\phi(r)}{(r-\xi)^{1-\sigma}} d r
$$

where $a+1>\lambda>\sigma, 0<\sigma<1, d<x<e$,

is a known function and

$$
F(x)=\frac{\Gamma(\sigma)}{\Gamma(1-\sigma)} \frac{d}{d x} \int_{d}^{x} \frac{\rho^{\lambda-1} f(\rho)}{(x-\rho)^{\sigma}} d \rho,
$$

$$
l(x, \xi)=\frac{d}{d x} \int_{d}^{x} \frac{d \rho}{(x-\rho)^{\sigma}(\rho-\xi)^{1-\sigma}} .
$$

It can easily be shown that

$$
l(x, \xi)=\frac{(d-\xi)^{\sigma}}{(x-\xi)(x-d)^{\sigma}}, \quad 0<\sigma<1,
$$

and hence equation (26) becomes

$$
m(x) \Phi(x)=F(x)-\frac{\sin (\sigma \pi)}{\pi(x-d)^{\sigma}} \int_{0}^{d} \frac{m(\xi)(d-\xi)^{\sigma}}{x-\xi} d \xi \int_{d}^{e} \frac{\phi(r)}{(r-\xi)^{1-\sigma}} d r .
$$

Using equation (25) we can write the last integral in the above equation as

$$
\begin{aligned}
& \int_{d}^{e} \frac{\phi(r)}{(r-\xi)^{1-\sigma}} d r=-\frac{\sin (\sigma \pi)}{\pi} \int_{d}^{e} \frac{d r}{(r-\xi)^{1-\sigma}} \frac{d}{d r} \int_{r}^{e} \frac{\Phi(x)}{(x-r)^{\sigma}} d x \\
& =\frac{\sin (\sigma \pi)}{\pi}\left\{(d-\xi)^{\sigma-1} \int_{d}^{e} \frac{\Phi(x)}{(x-d)^{\sigma}} d x-(1-\sigma) \int_{d}^{e} \frac{d r}{(r-\xi)^{2-\sigma}} \int_{r}^{e} \frac{\Phi(x)}{(x-r)^{\sigma}} d x\right\},
\end{aligned}
$$

after an integration by parts. Inverting the order of integration in the last term of equation (31) and using the result

$$
(1-\sigma) \int_{d}^{x} \frac{d r}{(r-\xi)^{2-\sigma}(x-r)^{\sigma}}=\frac{(x-d)^{1-\sigma}}{(x-\xi)(d-\xi)^{1-\sigma}}, \quad 0<\sigma<1,
$$

we find that

$$
\int_{d}^{e} \frac{\phi(r)}{(r-\xi)^{1-\sigma}} d r=(d-\xi)^{\sigma} \frac{\sin (\sigma \pi)}{\pi} \int_{d}^{e} \frac{\Phi(x)}{(x-\xi)(x-d)^{\sigma}} d x .
$$

Substituting this expression into equation (30) we find that $\Phi(x)$ is given by the equation

$$
m(x) \Phi(x)+\int_{d}^{e} \Phi(y) S(x, y) d y=F(x), \quad d<x<e
$$

where

$$
S(x, y)=\frac{\sin ^{2}(\sigma \pi)}{\pi^{2}[(x-d)(y-d)]^{\sigma}} \int_{0}^{d} \frac{m(\xi)(d-\xi)^{2 \sigma}}{(x-\xi)(y-\xi)} d \xi,
$$

and $a+1>\lambda>\sigma, 0<\sigma<1$. 
Equation (34) is a Fredholm integral equation of the second kind from which $\Phi(x)$ can be determined, $\phi(r)$ is then found from equation (25) and the coefficients $C_{n}$, which satisfy equations (1), (2) and (3) with $g_{1}=h_{1}=0$ when $a+1>\lambda>\sigma, 0<\sigma<1$, can be obtained from equation (20). It is perhaps interesting to note that equations (34) and (35) are of the same form as those obtained by Cooke $(7$, p. 196).

\section{Equations of the second kind}

To solve the triple series equations of the second kind (with $f_{1}=0$ ) we introduce two functions $\psi_{1}(\rho)$ and $\psi_{2}(\rho)$ defined by the equations

$$
\begin{aligned}
\sum_{n=0}^{\infty} D_{n} J_{n}(a, \lambda ; \rho) & =(1-\rho)_{1}^{\lambda-a} \psi_{1}(\rho), \quad 0 \leqq \rho<d, \\
& =(1-\rho)^{\lambda-a} \psi_{2}(\rho), \quad e<\rho \leqq 1,
\end{aligned}
$$

and using the orthogonality relation (15) we obtain from equations (36), (5) and (37) the following expression for the coefficients $D_{n}$

$$
D_{n}=\Delta_{n}^{2}\left\{\int_{0}^{d} \psi_{1}(r)+\int_{e}^{1} \psi_{2}(r)\right\} r^{\lambda-1} J_{n}(a, \lambda ; r) d r, \quad a+1>\lambda>0 .
$$

After substituting for $D_{n}$ in equations (4) and (6) and interchanging the order of summation and integration we obtain the equations

$$
\begin{aligned}
& \begin{aligned}
\int_{0}^{\rho} \psi_{1}(r) k_{r}(r, \rho) d r+\int_{\rho}^{d} \psi_{1}(r) k_{\rho}(r, \rho) d r+ & \int_{e}^{1} \psi_{2}(r) k_{\rho}(r, \rho) d r \\
& =\{\Gamma(\sigma)\}^{2} \rho^{\lambda-1} g(\rho), \quad 0 \leqq \rho<d, \\
\int_{0}^{d} \psi_{1}(r) k_{r}(r, \rho) d r+\int_{e}^{\rho} \psi_{2}(r) k_{r}(r, \rho) d r+ & \int_{\rho}^{1} \psi_{2}(r) k_{\rho}(r, \rho) d r \\
& =\{\Gamma(\sigma)\}^{2} \rho^{\lambda-1} h(\rho), \quad e<\rho \leqq 1,
\end{aligned}
\end{aligned}
$$

where $a+1>\lambda>\sigma>0$ and $k_{t}(r, \rho)$ is defined by equation (18). become

Inverting the order of integration in the above equations we find that they

$$
\int_{0}^{\rho} \frac{m(x)}{(\rho-x)^{1-\sigma}}\left\{\Psi_{1}(x)+\int_{e}^{1} \frac{\psi_{2}(r)}{(r-x)^{1-\sigma}} d r\right\} d x=\{\Gamma(\sigma)\}^{2} \rho^{\lambda-1} g(\rho),
$$

when $0 \leqq \rho<d$,

$$
\begin{aligned}
\int_{e}^{\rho} \frac{m(x)}{(\rho-x)^{1-\sigma}} \Psi_{2}(x) d x=\{\Gamma(\sigma)\}^{2} \rho^{\lambda-1} h(\rho)- & \int_{0}^{d} \frac{m(x)}{(\rho-x)^{1-\sigma}} \Psi_{1}(x) d x \\
& -\int_{0}^{e} \frac{m(x)}{(\rho-x)^{1-\sigma}} d x \int_{e}^{1} \frac{\psi_{2}(r)}{(r-x)^{1-\sigma}} d r,
\end{aligned}
$$


where $e<\rho \leqq 1$, and

$$
\text { (a) } \Psi_{1}(x)=\int_{x}^{d} \frac{\psi_{1}(r)}{(r-x)^{1-\sigma}} d r, \quad(b) \Psi_{2}(x)=\int_{x}^{1} \frac{\psi_{2}(r)}{(r-x)^{1-\sigma}} d r .
$$

When $0<\sigma<1$ we can solve the Abel-type integral equations (41), (42) and (43) and obtain, in a similar way to that used to obtain equations (25) and (30), the equations

$$
\begin{gathered}
m(x) \Psi_{1}(x)=G(x)-m(x) \int_{e}^{1} \frac{\psi_{2}(r)}{(r-x)^{1-\sigma}} d r, \quad 0<x<d, \\
m(x) \Psi_{2}(x)=H(x)-\frac{\sin (\sigma \pi)}{\pi(x-e)^{\sigma}} \int_{0}^{d} \frac{m(\xi)(e-\xi)^{\sigma}}{x-\xi} \Psi_{1}(\xi) d \xi \\
\quad-\frac{\sin (\sigma \pi)}{\pi(x-e)^{\sigma}} \int_{0}^{e} \frac{m(\xi)(e-\xi)^{\sigma}}{x-\xi} d \xi \int_{e}^{1} \frac{\psi_{2}(r)}{(r-\xi)^{1-\sigma}} d r,
\end{gathered}
$$

where $e<x<1$,

$$
\begin{array}{ll}
\psi_{1}(r)=-\frac{\sin (\sigma \pi)}{\pi} \frac{d}{d r} \int_{r}^{d} \frac{\Psi_{1}(x)}{(x-r)^{\sigma}} d x, & 0<r<d, \\
\psi_{2}(r)=-\frac{\sin (\sigma \pi)}{\pi} \frac{d}{d r} \int_{r}^{1} \frac{\Psi_{2}(x)}{(x-r)^{\sigma}} d x, & e<r<1,
\end{array}
$$

and the known functions $G(x)$ and $H(x)$ are given by

$$
\begin{array}{ll}
G(x)=\frac{\Gamma(\sigma)}{\Gamma(1-\sigma)} \frac{d}{d x} \int_{0}^{x} \frac{\rho^{\lambda-1} g(\rho)}{(x-\rho)^{\sigma}} d \rho, & 0<x<d, \\
H(x)=\frac{\Gamma(\sigma)}{\Gamma(1-\sigma)} \frac{d}{d x} \int_{e}^{x} \frac{\rho^{\lambda-1} h(\rho)}{(x-\rho)^{\sigma}} d \rho, & e<x<1 .
\end{array}
$$

From equations (44) and (46) we can show that the functions $\psi_{1}(r)$ and $\psi_{2}(r)$ are related by the equation

$$
\begin{aligned}
\psi_{1}(r)=-\frac{\sin (\sigma \pi)}{\pi} \frac{d}{d r} \int_{r}^{d} \frac{G(x)}{m(x)(x-r)^{\sigma}} d x \\
\quad-\frac{\sin (\sigma \pi)}{\pi(d-r)^{\sigma}} \int_{e}^{1} \frac{(\xi-d)^{\sigma}}{\xi-r} \psi_{2}(\xi) d \xi, \quad 0<r<d .
\end{aligned}
$$

By a method similar to that used to obtain equation (33) we can show that

$$
\int_{e}^{1} \frac{\psi_{2}(r)}{(r-\xi)^{1-\sigma}} d r=(e-\xi)^{\sigma} \frac{\sin (\sigma \pi)}{\pi} \int_{e}^{1} \frac{\Psi_{2}(y)}{(y-\xi)(y-e)^{\sigma}} d y .
$$

Using this result together with equation (44) we see that equation (45) can be written in the form

$$
m(x) \Psi_{2}(x)+\int_{e}^{1} \Psi_{2}(y) T(x, y) d y=H(x)+G_{1}(x), \quad e<x<1,
$$


where

$$
T(x, y)=\frac{\sin ^{2}(\sigma \pi)}{\pi^{2}[(x-e)(y-e)]^{\sigma}} \int_{d}^{e} \frac{m(\xi)(e-\xi)^{2 \sigma}}{(x-\xi)(y-\xi)} d \xi,
$$

and $G_{1}(x)$ is the known function

$$
G_{1}(x)=-\frac{\sin (\sigma \pi)}{\pi(x-e)^{\sigma}} \int_{0}^{d} \frac{(e-\xi)^{\sigma}}{x-\xi} G(\xi) d \xi
$$

Equation (52) is a Fredholm integral equation of the second kind which determines $\Psi_{2}(x) ; \psi_{2}(r)$ can then be found from equation (47) and $\psi_{1}(r)$ from equation (50). Finally the coefficients $D_{n}$ which satisfy equations (4), (5) and (6) when $a+1>\lambda>\sigma, 0<\sigma<1$, can be obtained from equation (38).

\section{An application}

Putting $a=\lambda=1, \sigma=\frac{1}{2}, \rho=\sin ^{2} \theta / 2, \quad 0 \leqq \theta \leqq \pi, g_{1}=h_{1}=0$, in equations (1), (2) and (3) and using the relation $J_{n}\left(1,1 ; \sin ^{2} \theta / 2\right)=P_{n}(\cos \theta)$, where $P_{n}(\cos \theta)$ is the Legendre polynomial, we find that they reduce to the equations

$$
\begin{gathered}
\sum_{n=0}^{\infty}(2 n+1) A_{n} P_{n}(\cos \theta)=0, \quad 0 \leqq \theta<\alpha, \quad \beta<\theta \leqq \pi, \\
\sum_{n=0}^{\infty} A_{n} P_{n}(\cos \theta)=F(\theta), \quad \alpha<\theta<\beta,
\end{gathered}
$$

where $(2 n+1) A_{n}=2 C_{n}, F(\theta)=f\left(\sin ^{2} \theta / 2\right), d=\sin ^{2} \alpha / 2$ and $e=\sin ^{2} \beta / 2$. These are the equations solved by Collins (6) when considering the electrostatic potential problem for a spherical ring with semi-angles $\alpha$ and $\beta$.

Using the results (20), (25), (34) and (35) of Section 2 it can be shown that a solution of equations (55) and (56) is

$$
A_{n}=\int_{\alpha}^{\beta} \phi(\theta) P_{n}(\cos \theta) d \theta
$$

where $\phi(\theta)$ is given in terms of a function $\Phi(t)$ by

$$
\phi(\theta)=-\frac{1}{\pi} \frac{d}{d \theta} \int_{\theta}^{\beta} \frac{\Phi(t) \sin t}{(\cos \theta-\cos t)^{\frac{1}{2}}} d t,
$$

and $\Phi(t)$ is determined from the Fredholm integral equation of the second kind

$$
\Phi(t)+\int_{\alpha}^{\beta} \Phi(u) S(t, u) d u=\frac{d}{d t} \int_{\alpha}^{t} \frac{F(\lambda) \sin \lambda}{(\cos \lambda-\cos t)^{\frac{1}{2}}} d \lambda
$$


with the symmetric kernel

$$
\begin{aligned}
S(t, u)= & \frac{\sin t \sin u}{\pi^{2}[(\cos \alpha-\cos t)(\cos \alpha-\cos u)]^{\frac{1}{2}}} \int_{0}^{\alpha} \frac{(\cos \sigma-\cos \alpha)}{(\cos \sigma-\cos t)(\cos \sigma-\cos u)} d \sigma, \\
= & \frac{1}{\pi^{2}(\cos t-\cos u)}\left\{\sin t\left(\frac{\cos \alpha-\cos u}{\cos \alpha-\cos t}\right)^{\frac{1}{2}} \log \left[\frac{\sin (u+\alpha) / 2}{\sin (u-\alpha) / 2}\right]\right. \\
& \left.\quad-\sin u\left(\frac{\cos \alpha-\cos t}{\cos \alpha-\cos u}\right)^{\frac{1}{2}} \log \left[\frac{\sin (t+\alpha) / 2}{\sin (t-\alpha) / 2}\right]\right\} .
\end{aligned}
$$

Collins obtained the solution of equations (55) and (56) in terms of a pair of Fredholm integral equations of the second kind by a different method from that used in this paper. His integral equations however, can be considered to be more useful than equation (59) since they are amenable to iteration for small values of $\alpha$ or $\pi-\beta$.

\section{REFERENCES}

(1) B. Noble, Some dual series equations involving Jacobi polynomials, Proc. Camb. Phil. Soc. 59 (1963), 363.

(2) J. S. Lowndes, Dual series and triple integral equations, (to be published).

(3) W. MAGnus and F. OberhetTinger, Formulas and theorems for the functions of mathematical physics (Chelsea, 1954).

(4) A. ERDELYI et al., Higher transcendental functions (McGraw-Hill, 1953).

(5) K. N. SRIvastava, On triple series equations involving series of Jacobi polynomials, Proc. Edin. Math. Soc. 15 (1967), 221.

(6) W. D. Collns, On some triple series equations and their applications, Arch. Rat. Mech. Anal. 11 (1962) 122.

(7) J. C. Cooke, Triple integral equations, Quart. Journ. Mech. Appl. Math. 16 (1963), 193.

\section{UNIVERSITY OF STRATHCLYDE}

GLASGOW 